Supporting Information for:

\title{
Molecular Simulation Studies on the Interactions of 2,4,6-Trinitrotoluene and Its Metabolites with Lipid Membranes
}

\author{
Hong Yang, ${ }^{\dagger, \ddagger}, \|$ Huarong Li, ${ }^{\ddagger} \|$ Liu Liu, ${ }^{\ddagger}$ Yang Zhou, ${ }^{\ddagger, *}$ Xinping Long ${ }^{\ddagger}$ \\ † School of Material Science and Engineering, Tsinghua University, Beijing \\ 100084, China. \\ ₹ Institute of Chemical Materials, Chinese Academy of Engineering and \\ Physics, Mianyang 621900, China;
}

*To whom Correspondence should be addressed: zhouy@caep.cn (Y. Zhou) 
Table S1. Statistics on epidemiological survey results of TNT poisoning in China (prevalence rate, $\%$ )

\begin{tabular}{|c|c|c|c|c|c|}
\hline \multirow{2}{*}{ year } & \multirow{2}{*}{ area } & \multirow{2}{*}{ lens damage } & \multicolumn{3}{|c|}{ cataract } \\
\hline & & & I & II & III \\
\hline 1973 & China & & & 33.0 & \\
\hline 1986 & Baotou & & & 40.8 & \\
\hline 1988 & & 57.3 & & & \\
\hline 1993 & & 46.2 & & & \\
\hline 1998 & Qinghai & 40.3 & & & \\
\hline 2003 & & 35.1 & & & \\
\hline 2008 & & 37.2 & & & \\
\hline 1998 & Zhejiang & 83.9 & 38.7 & 25.8 & 19.4 \\
\hline 2000 & Xiangxi & 35.0 & 12.2 & 12.2 & 1.6 \\
\hline 2004 & & 19.4 & 6.5 & 1.6 & \\
\hline 2002 & Shangluo & 39.2 & 11.8 & 4.5 & 1.6 \\
\hline 2003 & Fujian & 39.2 & & 14.9 & \\
\hline 2004 & Guangxi & 37.3 & & & \\
\hline 2005 & Lianyuan & 24.9 & 12.9 & 4.4 & \\
\hline 2007 & Chongqing & 45.7 & 32.0 & 11.3 & 2.3 \\
\hline 2008 & Chenzhou & 24.8 & 5.2 & 4.6 & \\
\hline 2010 & Nanjing & 42.9 & 8.9 & 5.9 & 1.7 \\
\hline 2012 & Linyi & 13.0 & & & \\
\hline
\end{tabular}

*Data sources: Xiaomei Bao, et.al. Inner Mongolia Medical Journal, 1989, 9, 32 (In Chinese); Shaoyuan Ma, China Occupational Medicine, 2010, 37,449 (In Chinese); Chenglai Zhou, et.al. Occupation and Health, 2001, 17, 7 (In Chinese); Yang Hou, Practical Preventive Medicine Journal, 2006, 131688 (In Chinese); Donghong Huo, et.al. Journal of Environmental and Occupational Medicine, 2002, 19, 264 (In Chinese); Chunhua Han, et.al. Chinese Journal of Coal Industry Medicine, 2004, 7, 580 (In Chinese); Lin Lv, et.al. Guangxi Journal of Preventive Medicine, 2004, 10, 82 (In Chinese); Qichu Liu, et.al. Practical Preventive Medicine, 2006, 13, 381 (In Chinese); Shuquan Luo, et.al. Modern Preventive Medicine, 2007, 34, 2281 (In Chinese); Linghui Yao, et.al. China Tropical Medicine, 2008, 8, 174 (In Chinese); Chunhong Zhu, et.al. The Medical Journal of Industrial Enterprise, 2011, 24, 8 (In Chinese); Zhaoying Liu, et.al. Occupation and Health, 2013, 29, 2914 (In Chinese). 
Table S2. Summary of all simulations performed

\begin{tabular}{|c|c|c|c|c|c|}
\hline \multicolumn{2}{|c|}{ Simulation } & Duration (ns) & \multicolumn{2}{|c|}{ Simulation } & Duration (ns) \\
\hline \multicolumn{6}{|c|}{ Unconstrained MD } \\
\hline solute & No. of solute & & solute & No. of solute & \\
\hline $2 \mathrm{~A}$ & 20 & 120 & $26 \mathrm{DA}$ & 20 & 120 \\
\hline $2 \mathrm{~A}$ & 40 & 120 & $26 \mathrm{DA}$ & 40 & 120 \\
\hline $2 \mathrm{~A}$ & 60 & 240 & $26 \mathrm{DA}$ & 60 & 120 \\
\hline $4 \mathrm{~A}$ & 20 & 120 & $4 \mathrm{HA}$ & 20 & 120 \\
\hline $4 \mathrm{~A}$ & 40 & 120 & $4 \mathrm{HA}$ & 40 & 120 \\
\hline $4 \mathrm{~A}$ & 60 & 240 & $4 \mathrm{HA}$ & 60 & 120 \\
\hline $24 \mathrm{DA}$ & 20 & 120 & TNT & 20 & 120 \\
\hline $24 \mathrm{DA}$ & 40 & 120 & TNT & 40 & 120 \\
\hline $24 \mathrm{DA}$ & 60 & 120 & TNT & 60 & 120 \\
\hline \multicolumn{6}{|c|}{ Constrained MD } \\
\hline & -ADNT & $50 \times 32$ & & & \\
\hline & -ADNT & $50 \times 32$ & & & \\
\hline & 4-DANT & $50 \times 32$ & & & \\
\hline & 6-DANT & $50 \times 32$ & & & \\
\hline & HA-DNT & $50 \times 32$ & & & \\
\hline
\end{tabular}

Table S3. The thickness, area per lipid, average order parameter $\left\langle S_{\mathrm{CD}}\right\rangle$ and lateral diffusion coefficient (D) of POPC membrane at different concentrations of TNT and its reduced metabolites.

\begin{tabular}{|c|c|c|c|c|c|}
\hline \multicolumn{2}{|c|}{ System } & \multirow[b]{2}{*}{ Thickness (nm) } & \multirow{2}{*}{$\begin{array}{c}\text { Area per lipid } \\
\qquad\left(\AA^{2}\right)\end{array}$} & \multirow[b]{2}{*}{$\left\langle S_{\mathrm{CD}}\right\rangle$} & \multirow{2}{*}{$\begin{array}{c}\text { D }\left(\times 10^{-7}\right. \\
\left.\mathbf{c m}^{2} / \mathbf{s}\right)\end{array}$} \\
\hline solute & $\begin{array}{l}\text { No. of } \\
\text { solute }\end{array}$ & & & & \\
\hline \multicolumn{2}{|c|}{ POPC } & $3.89 \pm 0.06$ & $64.7 \pm 1.2$ & $0.152 \pm 0.01$ & $0.91 \pm 0.07$ \\
\hline \multirow{3}{*}{ TNT } & 20 & $3.84 \pm 0.05$ & $66.9 \pm 1.1$ & $0.154 \pm 0.02$ & $0.82 \pm 0.06$ \\
\hline & 40 & $3.84 \pm 0.05$ & $68.9 \pm 1.1$ & $0.153 \pm 0.05$ & $0.61 \pm 0.04$ \\
\hline & 60 & $3.80 \pm 0.05$ & $71.8 \pm 1.1$ & $0.146 \pm 0.04$ & $0.63 \pm 0.01$ \\
\hline \multirow{3}{*}{$2 \mathrm{~A}$} & 20 & $3.87 \pm 0.06$ & $66.2 \pm 1.3$ & $0.156 \pm 0.02$ & $1.11 \pm 0.03$ \\
\hline & 40 & $3.78 \pm 0.07$ & $69.8 \pm 1.5$ & $0.148 \pm 0.01$ & $0.73 \pm 0.04$ \\
\hline & 60 & $3.78 \pm 0.07$ & $71.4 \pm 1.5$ & $0.148 \pm 0.01$ & $0.70 \pm 0.02$ \\
\hline \multirow{3}{*}{$4 \mathrm{~A}$} & 20 & $3.86 \pm 0.06$ & $66.5 \pm 1.2$ & $0.152 \pm 0.01$ & $1.05 \pm 0.04$ \\
\hline & 40 & $3.75 \pm 0.06$ & $70.5 \pm 1.3$ & $0.145 \pm 0.02$ & $0.68 \pm 0.03$ \\
\hline & 60 & $3.76 \pm 0.06$ & $71.7 \pm 1.4$ & $0.146 \pm 0.02$ & $0.77 \pm 0.01$ \\
\hline \multirow{3}{*}{ 24DA } & 20 & $3.86 \pm 0.07$ & $66.3 \pm 1.5$ & $0.150 \pm 0.01$ & $1.04 \pm 0.06$ \\
\hline & 40 & $3.80 \pm 0.05$ & $68.5 \pm 1.0$ & $0.146 \pm 0.02$ & $1.15 \pm 0.03$ \\
\hline & 60 & $3.69 \pm 0.05$ & $71.8 \pm 1.1$ & $0.138 \pm 0.02$ & $0.75 \pm 0.09$ \\
\hline \multirow{3}{*}{ 26DA } & 20 & $3.88 \pm 0.06$ & $65.4 \pm 1.3$ & $0.151 \pm 0.01$ & $1.13 \pm 0.01$ \\
\hline & 40 & $3.83 \pm 0.06$ & $67.1 \pm 1.4$ & $0.146 \pm 0.04$ & $0.99 \pm 0.02$ \\
\hline & 60 & $3.75 \pm 0.06$ & $70.0 \pm 1.5$ & $0.143 \pm 0.04$ & $1.01 \pm 0.03$ \\
\hline \multirow{3}{*}{$4 \mathrm{HA}$} & 20 & $3.88 \pm 0.06$ & $66.4 \pm 1.2$ & $0.156 \pm 0.01$ & $0.76 \pm 0.04$ \\
\hline & 40 & $3.80 \pm 0.05$ & $69.7 \pm 1.1$ & $0.146 \pm 0.06$ & $0.76 \pm 0.03$ \\
\hline & 60 & $3.68 \pm 0.06$ & $73.8 \pm 1.5$ & $0.144 \pm 0.03$ & $0.66 \pm 0.05$ \\
\hline
\end{tabular}




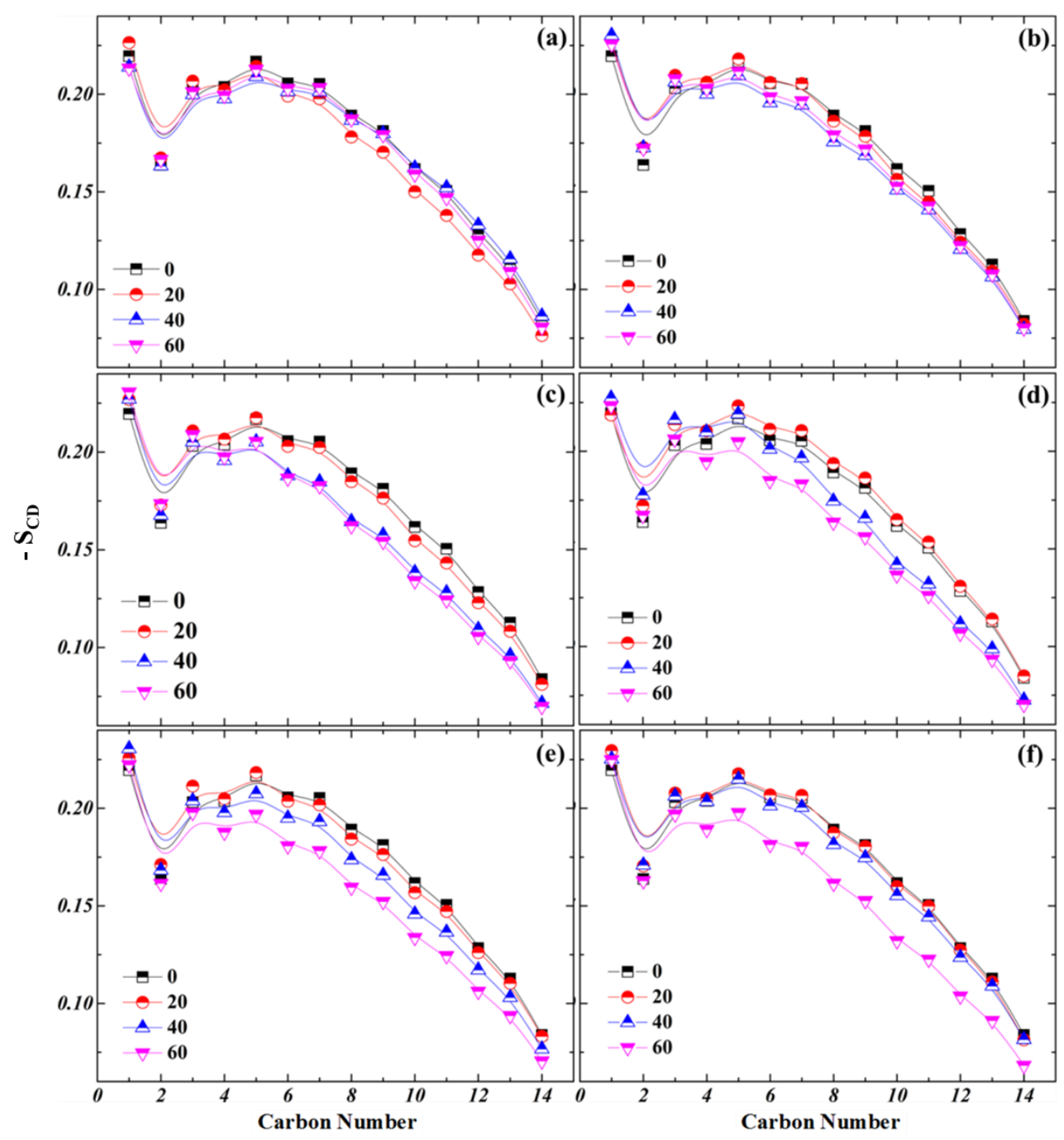

Figure S1. Deuterium order parameters in the $s n-1$ chains of POPC lipids at different concentration. (a) TNT, (b) 2A, (c) 4A, (d) 4HA, (e) 24DA, (f) 26DA. 

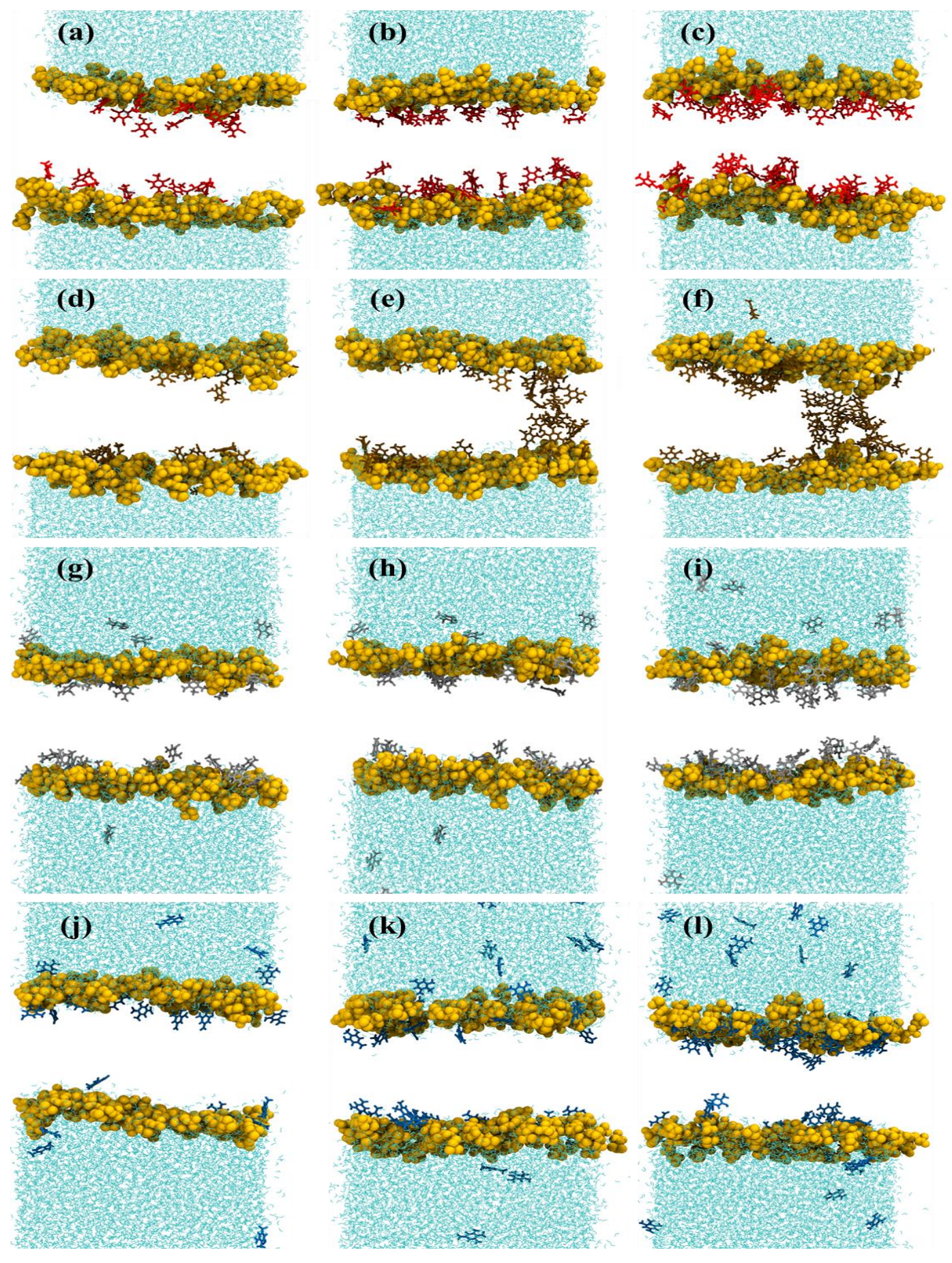

(h)
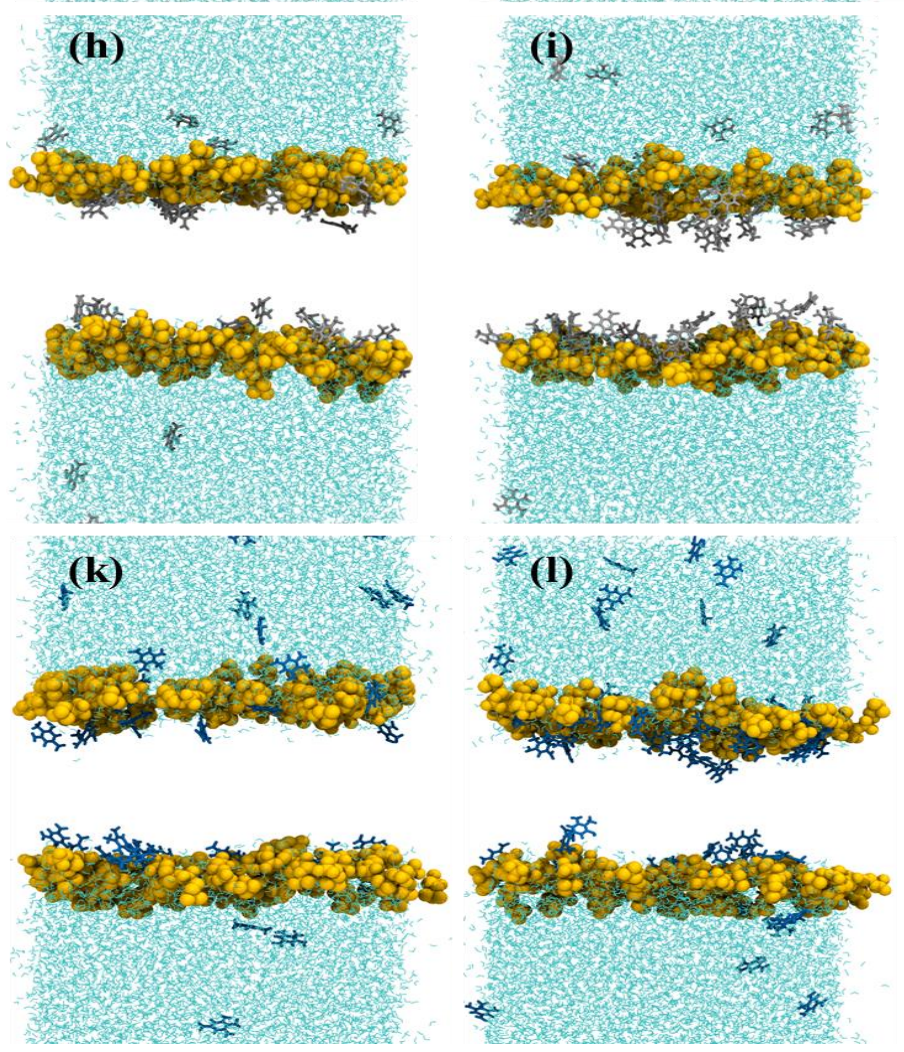

Figure S2. Final snapshots of (a) 20 4HA, (b) 40 4HA, (c) 60 4HA, (d) 20 TNT, (e) 40 TNT, (f) 60 TNT, (g) 20 24DA, (h) 40 24DA, (i) 60 24DA, (j) 20 26DA, (k) 40 26DA, (1) 60 26DA system. The lipid tails are omitted for clarify. The color rules are: lipid heads (yellow), water (cyan), 4HA (red), TNT (ochre) 24DA (silver) 26DA (blue). The periodicity is removed to maintain the integrity of molecules. 


\section{Parameters and structure of 4HA (NHOH)}

\section{NHOH.itp}

; Created by cgenff_charmm2gmx.py

[ moleculetype ]

; Name nrexcl

nhoh

3

[ atoms ]

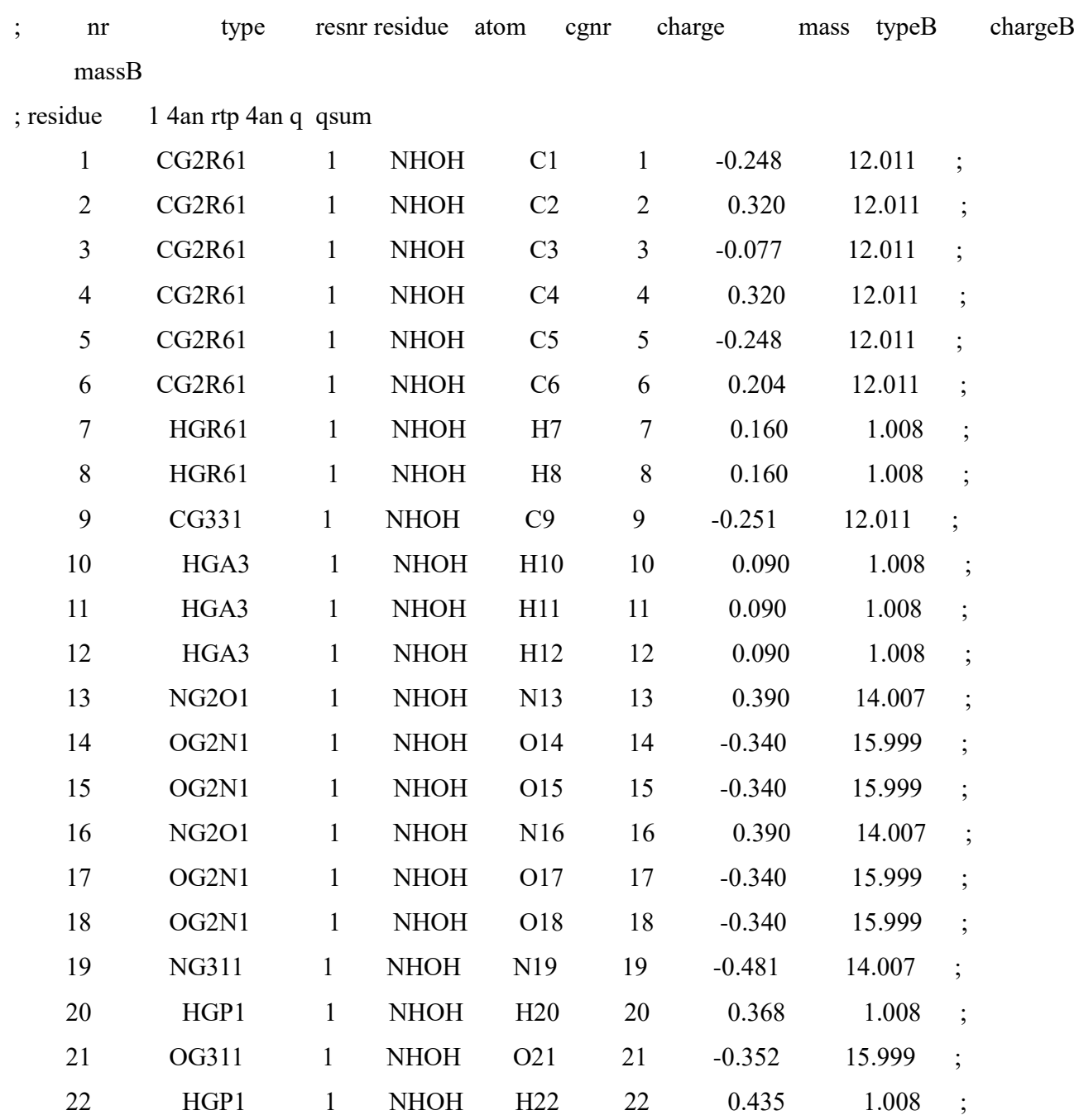

[bonds ]

$\begin{array}{ccccccc}; & \text { ai } & \text { aj funct } & \text { c0 } & \text { c1 } & \text { c2 } \\ 1 & 2 & 1 & & \\ 1 & 6 & 1 & & \\ 1 & 7 & 1 & & \\ 2 & 3 & 1 & & \\ 2 & 16 & 1 & & \end{array}$




$\begin{array}{rrr}3 & 9 & 1 \\ 3 & 4 & 1 \\ 4 & 13 & 1 \\ 4 & 5 & 1 \\ 5 & 6 & 1 \\ 5 & 8 & 1 \\ 6 & 19 & 1 \\ 9 & 10 & 1 \\ 9 & 12 & 1 \\ 9 & 11 & 1 \\ 13 & 14 & 1 \\ 13 & 15 & 1 \\ 16 & 17 & 1 \\ 16 & 18 & 1 \\ 19 & 20 & 1 \\ 19 & 21 & 1 \\ 21 & 22 & 1\end{array}$

[ pairs ]

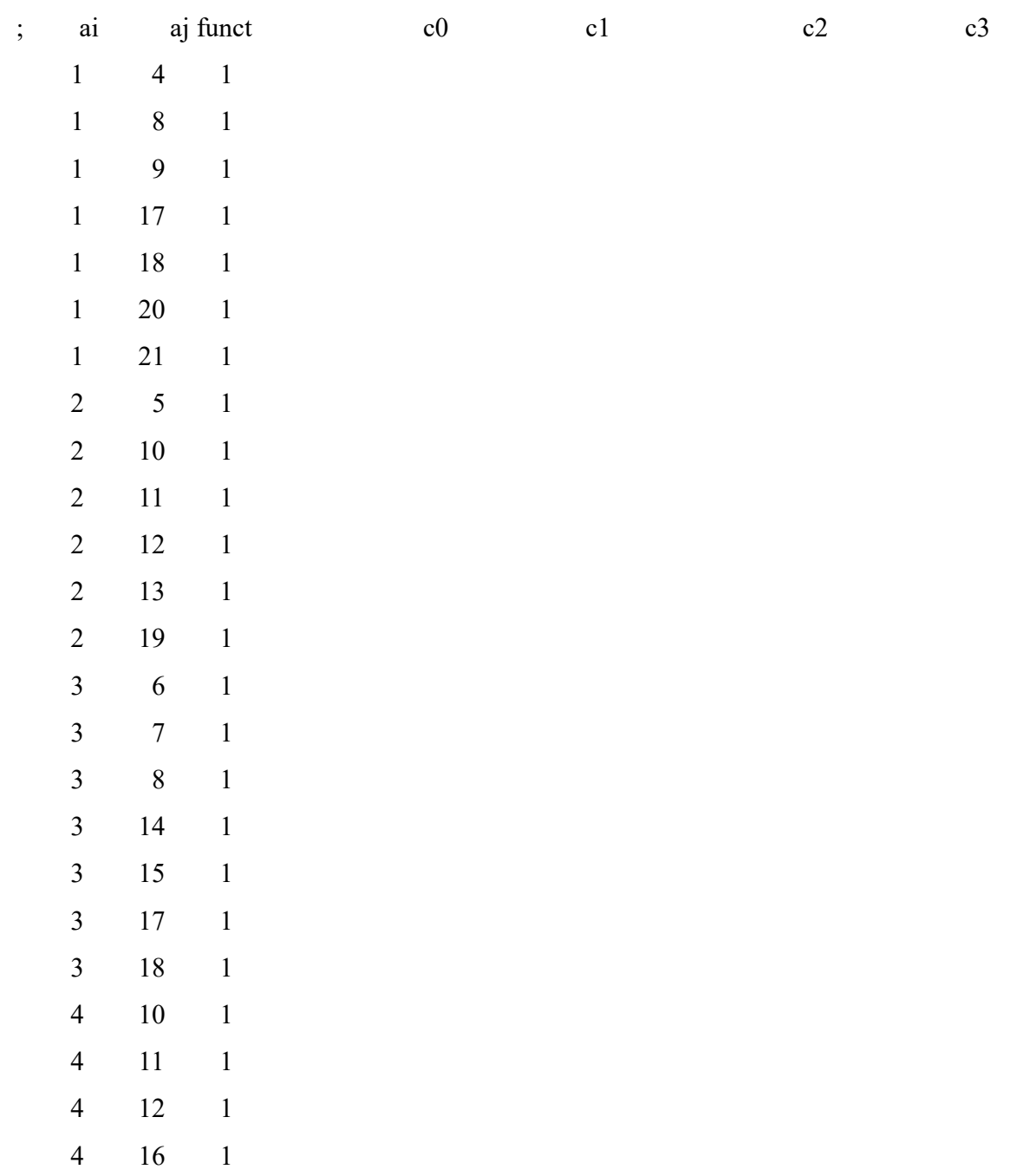




$\begin{array}{rrr}4 & 19 & 1 \\ 5 & 7 & 1 \\ 5 & 9 & 1 \\ 5 & 14 & 1 \\ 5 & 15 & 1 \\ 5 & 20 & 1 \\ 5 & 21 & 1 \\ 6 & 13 & 1 \\ 6 & 22 & 1 \\ 6 & 16 & 1 \\ 7 & 19 & 1 \\ 7 & 16 & 1 \\ 8 & 13 & 1 \\ 8 & 19 & 1 \\ 9 & 13 & 1 \\ 9 & 16 & 1 \\ 20 & 22 & 1\end{array}$

[ angles ]

$\begin{array}{rrrrr}\text { ai } & \text { aj } & \text { ak funct } & \text { c0 } \\ 2 & 1 & 6 & 5 \\ 2 & 1 & 7 & 5 \\ 6 & 1 & 7 & 5 \\ 1 & 2 & 3 & 5 \\ 1 & 2 & 16 & 5 \\ 3 & 2 & 16 & 5 \\ 9 & 3 & 2 & 5 \\ 9 & 3 & 4 & 5 \\ 2 & 3 & 4 & 5 \\ 13 & 4 & 3 & 5 \\ 13 & 4 & 5 & 5 \\ 3 & 4 & 5 & 5 \\ 4 & 5 & 6 & 5 \\ 4 & 5 & 8 & 5 \\ 6 & 5 & 8 & 5 \\ 1 & 6 & 19 & 5 \\ 1 & 6 & 5 & 5 \\ 19 & 6 & 5 & 5 \\ 10 & 9 & 3 & 5 \\ 10 & 9 & 12 & 5 \\ 3 & 9 & 11 & 5 \\ 12 & 9 & 11 & 5\end{array}$




$\begin{array}{rrrr}4 & 13 & 14 & 5 \\ 4 & 13 & 15 & 5 \\ 14 & 13 & 15 & 5 \\ 17 & 16 & 2 & 5 \\ 17 & 16 & 18 & 5 \\ 2 & 16 & 18 & 5 \\ 20 & 19 & 21 & 5 \\ 20 & 19 & 6 & 5 \\ 21 & 19 & 6 & 5 \\ 19 & 21 & 22 & 5\end{array}$

[ dihedrals ]

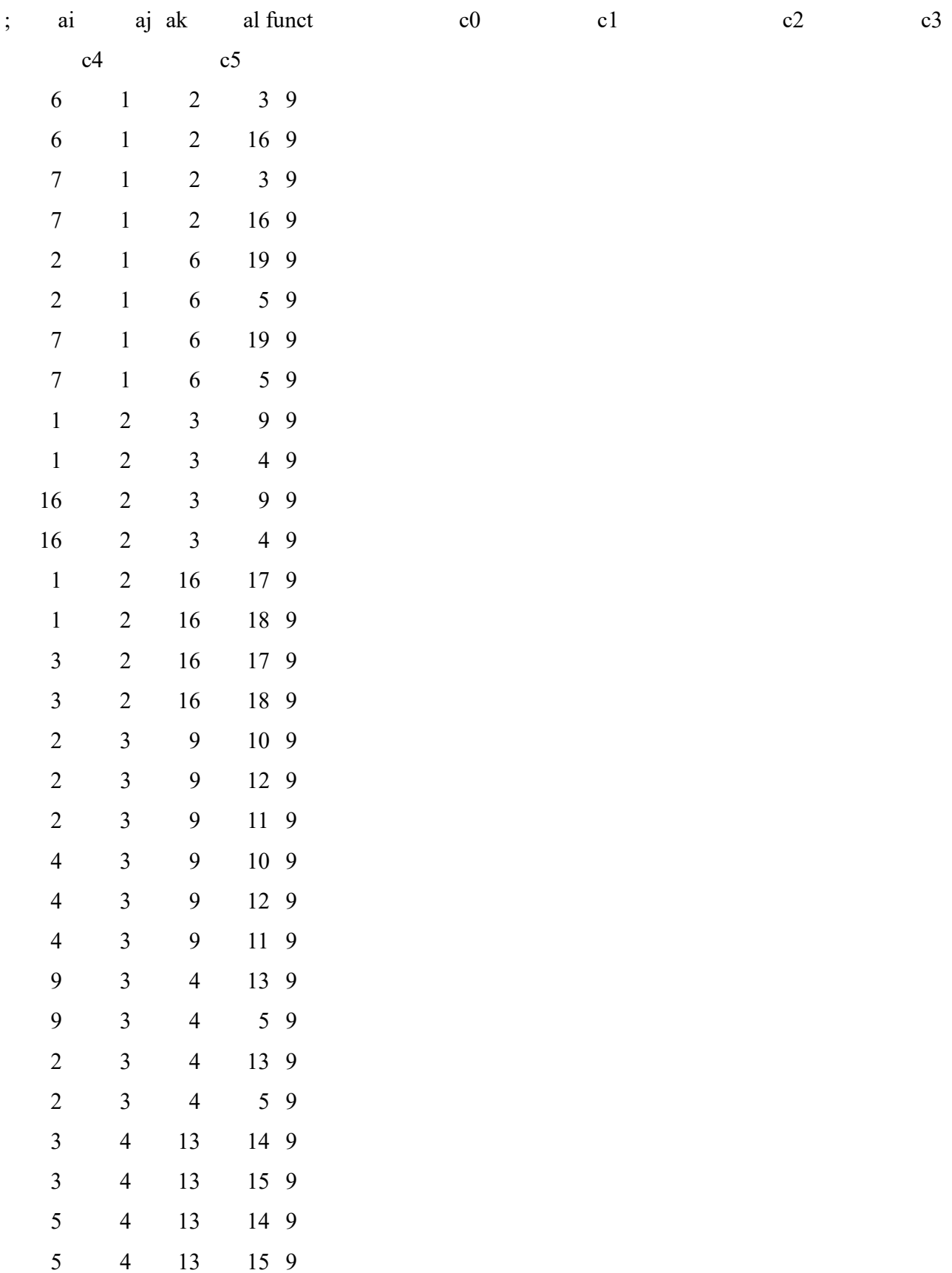




$\begin{array}{rrrrr}13 & 4 & 5 & 6 & 9 \\ 13 & 4 & 5 & 8 & 9 \\ 3 & 4 & 5 & 6 & 9 \\ 3 & 4 & 5 & 8 & 9 \\ 4 & 5 & 6 & 1 & 9 \\ 4 & 5 & 6 & 19 & 9 \\ 8 & 5 & 6 & 1 & 9 \\ 8 & 5 & 6 & 19 & 9 \\ 1 & 6 & 19 & 20 & 9 \\ 1 & 6 & 19 & 21 & 9 \\ 5 & 6 & 19 & 20 & 9 \\ 5 & 6 & 19 & 21 & 9 \\ 20 & 19 & 21 & 22 & 9 \\ 6 & 19 & 21 & 22 & 9\end{array}$

\section{NHOH.prm}

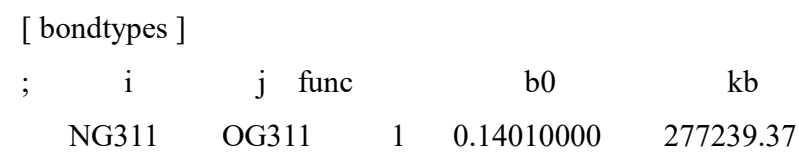

\begin{tabular}{rccccrrr}
; & j & k & l func & \multicolumn{1}{c}{ phi0 } & kphi & mult \\
CG2R61 & CG2R61 & NG311 & OG311 & 9 & 0.000000 & 49.258232 & 1 \\
CG2R61 & CG2R61 & NG311 & OG311 & 9 & 180.000000 & 8.229928 & 2 \\
CG2R61 & CG2R61 & NG311 & OG311 & 9 & 0.000000 & 6.259264 & 3 \\
CG331 & CG2R61 & CG2R61 & NG2O1 & 9 & 180.000000 & 23.68144 & 2 \\
CG331 & CG2R61 & CG2R61 & NG2O1 & 9 & 0.000000 & 5.4392 & 3 \\
CG2R61 & NG311 & OG311 & HGP1 & 9 & 180.000000 & 11.614784 & 2 \\
HGP1 & NG311 & OG311 & HGP1 & 9 & 180.000000 & 7.497728 & 1 \\
HGP1 & NG311 & OG311 & HGP1 & 9 & 0.000000 & 12.710992 & 2 \\
HGP1 & NG311 & OG311 & HGP1 & 9 & 0.000000 & 3.112896 & 3
\end{tabular}

[ dihedraltypes ] 
; 'improper' dihedrals

; $\quad \mathrm{i} \quad \mathrm{j} \quad \mathrm{k} \quad 1$ func $\quad$ phi0

\section{NHOH.pdb}

\begin{tabular}{|c|c|c|c|c|c|c|c|}
\hline ATOM & $1 \mathrm{C} 1$ nhoh & 1 & 1.519 & -0.256 & -0.072 & 1.00 & 0.00 \\
\hline ATOM & $2 \mathrm{C} 2$ nhoh & 1 & 0.716 & 0.879 & 0.005 & 1.00 & 0.00 \\
\hline ATOM & $3 \mathrm{C} 3$ nhoh & 1 & -0.691 & 0.863 & 0.060 & 1.00 & 0.00 \\
\hline ATOM & $4 \mathrm{C} 4$ nhoh & 1 & -1.229 & -0.439 & 0.006 & 1.00 & 0.00 \\
\hline ATOM & 5 C5 nhoh & 1 & -0.467 & -1.600 & -0.069 & 1.00 & 0.00 \\
\hline ATOM & 6C6 nhoh & 1 & 0.926 & -1.518 & -0.121 & 1.00 & 0.00 \\
\hline ATOM & $7 \mathrm{H} 7$ nhoh & 1 & 2.594 & -0.151 & -0.074 & 1.00 & 0.00 \\
\hline ATOM & $8 \mathrm{H} 8$ nhoh & 1 & -0.979 & -2.555 & -0.089 & 1.00 & 0.00 \\
\hline ATOM & 9 C9 nhoh & 1 & -1.531 & 2.103 & 0.258 & 1.00 & 0.00 \\
\hline ATOM & $10 \mathrm{H} 10$ nhoh & 1 & -1.834 & 2.526 & -0.703 & 1.00 & 0.00 \\
\hline ATOM & $11 \mathrm{H} 11$ nhoh & 1 & -2.440 & 1.872 & 0.812 & 1.00 & 0.00 \\
\hline ATOM & $12 \mathrm{H} 12$ nhoh & 1 & -0.970 & 2.871 & 0.790 & 1.00 & 0.00 \\
\hline ATOM & 13 N13 nhoh & 1 & -2.691 & -0.661 & 0.016 & 1.00 & 0.00 \\
\hline ATOM & 14 O14 nhoh & 1 & -3.098 & -1.714 & 0.508 & 1.00 & 0.00 \\
\hline ATOM & 15 O15 nhoh & 1 & -3.409 & 0.196 & -0.493 & 1.00 & 0.00 \\
\hline ATOM & 16 N16 nhoh & 1 & 1.459 & 2.159 & 0.012 & 1.00 & 0.00 \\
\hline ATOM & 17 O17 nhoh & 1 & 0.935 & 3.130 & -0.530 & 1.00 & 0.00 \\
\hline ATOM & $18 \mathrm{O} 18$ nhoh & 1 & 2.573 & 2.162 & 0.532 & 1.00 & 0.00 \\
\hline ATOM & 19 N19 nhoh & 1 & 1.681 & -2.690 & -0.302 & 1.00 & 0.00 \\
\hline ATOM & $20 \mathrm{H} 20$ nhoh & 1 & 1.287 & -3.481 & 0.203 & 1.00 & 0.00 \\
\hline ATOM & $21 \mathrm{O} 21 \mathrm{nhoh}$ & 1 & 3.025 & -2.551 & 0.137 & 1.00 & 0.00 \\
\hline ATOM & $22 \mathrm{H} 22$ nhoh & 1 & 3.538 & -2.721 & -0.667 & 1.00 & 0.00 \\
\hline
\end{tabular}

